\title{
A NEW AGENT IN PREVENTION OF CONTRAST-INDUCED DRAGON FRUIT NEPHROPATHY: (HYLOCEREUS POLYRHIZUS) EXTRACT
}

\section{Levent Sahin 1 , Erdem Toktay², Ahmet Harmankaya ${ }^{3}$, Pinar Aksu Kilicle ${ }^{4}$, Muhammed Yayla ${ }^{5}$, Huseyin Fatih Gul ${ }^{6}$}

\footnotetext{
${ }^{1}$ Kafkas University, Faculty of Medicine, Department of Emergency Medicine, Kars, Turkey

${ }^{2}$ Kafkas University, Faculty of Medicine, Department of Histology and Embryology, Kars, Turkey

${ }^{3}$ Kafkas University, Faculty of Art and Science, Department of Chemistry, Kars, Turkey

${ }^{4}$ Kafkas University, Faculty of Art and Science, Department of Biology, Kars, Turkey

${ }^{5}$ Kafkas University, Faculty of Medicine, Department of Pharmacology, Kars, Turkey

${ }^{6}$ Kafkas University, Faculty of Medicine, Department of Biochemistry, Kars, Turkey
}

Address for Correspondence: Assoc.Prof. Levent Sahin, E-mail: levsahin44@gmail.com Received: 28.05.2021; Accepted: 16.08.2021; Available Online Date: 20.09.2021

(C) Copyright 2021 by Dokuz Eylül University, Institute of Health Sciences - Available online at https://dergipark.org.tr/en/pub/jbachs

Cite this article as: Sahin L, Toktay E, Harmankaya A, Aksu-Kilicle P, Yayla M, Gul HF. A New Agent in Prevention of Contrast-Induced Nephropathy: Dragon Fruit (Hylocereus Polyrhizus) Extract. J Basic Clin Health Sci 2021; 3: 117- 125.

\begin{abstract}
Purpose: We aimed to investigate dragon fruit (Hylocereus polyrhizus) extract effectiveness by creating a nephropathy model with contrast media toxicity after dehydration and furosemide administration to rats.

Methods: Total 36 male Sprague dawley rats were randomly allocated into 6 groups ( $n: 6)$. At the end of the experiment kidney organs and blood was taken for histopathologic and biochemical analyze (malondialdehyde, glutathione, serum urea nitrogen and serum creatine).

Results: Renal injury markers of serum urea nitrogen and creatinine levels decreased $(p<0.05)$ in the groups that given dragon fruit extract depending on the dose manner compared to the group that was given contrast media. While oxidative stress increased in given contrast media group, MDA levels improved statistically significance in the groups given dragon fruit extract depending on the dose compared to the group that was given contrast media. Also tissue GSH levels increased in the groups given dragon fruit extract depending on the dose compared to the group that was given contrast media. Vacuolization damage was minimal and inflammation was not seen in these groups according to the histopathological analyses.
\end{abstract}

Conclusion: Dragon fruit extract, a strong antioxidative agent, application together with contrast media can play a protective role against renal tubule via reduced renal injury markers, oxidative damage.

Keywords: Contrast induced nephropathy, dragon fruit extract, kidney, oxidative stress, rat

\section{INTRODUCTION}

Acute kidney injury (AKI) is a syndrome that results in the inability to remove nitrogenous waste products from the body due to the sudden loss of function in the kidney, and the deterioration of electrolyte balance (1). Dehydration, obstructions in the renal vessels, infections, drugs and the use of contrast can cause AKI. There is also an increase in the frequency of contrast-induced nephropathy (CIN) due to increased imaging techniques and interventional radiological procedures (2). CIN is the event of deterioration of renal functions with an increase of 
0.5-1.0 $\mathrm{mg} / \mathrm{dL}$ in serum creatinine value, $25 \%$ increase in basal creatinine and $25 \%$ decrease in glomerular filtration rate, after exposure to contrast (3).

The most common pathogenetic mechanisms in the development of nephrotoxicity are apoptosis that damage caused by oxidative stress, medullary hypoxia due to decrease in renal blood flow, inflammation and direct cellular toxicity (4). After administration of contrast, the production of free radicals increases through post-ischemic oxidative stress. Increased free radicals play a role in apoptosis of renal tubular and glomerular cells (5). Nephropathy results from necrotic and apoptotic cell death due to these mechanisms at the cellular level (6).

The primary aim has eliminated of renal tubule damage resulting from increasing CIN cases. While saline hydration is suggested to protect the kidneys from this damage, it is recommended to avoid hydration with high salty, high volume contrast agents and nephrotoxic drugs (7). The use of ascorbic acid, $\mathrm{N}$-acetylcysteine and sodium bicarbonate is recommended for protection from CIN (8-10). In studies, different pharmacological agents were also preferred to prevent CIN (11-13). In addition, the consumption of foods containing high antioxidants is also recommended for patients (7). Although some treatment strategies are being implemented in the clinic, there is still no definitive treatment for contrast nephropathy. This is why new studies are needed to prevent and treat contrast nephropathy.

Dragon fruit (Hylocereus polyrhizus) or red pitaya has high antioxidant capacity due to the large number of phenolic compounds it contains. Studies have also shown that the fruit has anti-inflammatory, antiproliferation, and highly antiradical due to the content of phytoalbumin (14-17). Red dragon fruit extract also has anticancer, hypocholesterolemic, and prebiotic effects (18). In the content analysis of dragon fruit extract in the literature, it was revealed that this fruit is rich in high isoflovoid and phenolic compounds, and its antioxidant properties were emphasized (19).

We thought that dragon fruit extract (DFE) could be an alternative treatment for kidney damage. We aimed to investigate its effectiveness on CIN by creating a nephropathy model with contrast media (CM) toxicity after dehydration and furosemide administration to rats. To demonstrate this effect, we examined blood urea nitrogen (BUN) and serum creatinine (SCR) levels, glutathione $(\mathrm{GSH})$ and malondialdehyde (MDA) levels, which play a role in CIN pathogenesis.

\section{METHODS}

\section{Ethics statement and animals}

The Local Ethics Committee of Animal Experiments of Kafkas University approved this study (2020/047). 36 Sprague dawley male rats with an average weight of 200-250 grams and 10-12 weeks old were obtained from Kafkas University experimental research and Application Center. The rats were given enough (ad libitum) water and pellet feed during the experiment. The animals were housed at optimal room temperature and humidity level.

\section{Extraction}

Dragon fruits were obtained from Mersin/Erdemli, Dragon Fruit Greenhouse. The peel of the fruit was peeled and the parts eaten as fruit were sliced. It was dried sparsely on blotter paper in an environment free from direct sunlight and provided with dry air flow. The dried fruit slices were ground in a grinder. The ground fruits were weighed with $0.1 \mathrm{mg}$ sensitivity and approximately 50 grams were taken into the cartridge washed with extraction solvent. The cartridge was placed in $500 \mathrm{~mL}$ Soxhlet extractor and $650 \mathrm{~mL}$ of solvent was added to the boiling flask. The solvent was extracted (9-12 siphons) until it was clear. $96 \%$ ethanol was used as the extraction solvent. After the obtained extract was filtered through the blue band filter paper and its particles were removed, the solvents were evaporated at $40-50^{\circ} \mathrm{C}$ with a rotary evaporator in the balloons brought to constant weight.

\section{Surgical procedures, Animal experimantal groups and Models}

The animals were randomly divided into 6 groups ( $\mathrm{n}$ : 6 ). No procedure was applied to the CONTROL group. Animals were left to dehydration for 48 hours before CM (lohexol, Kocsel, Turkey) was administered.

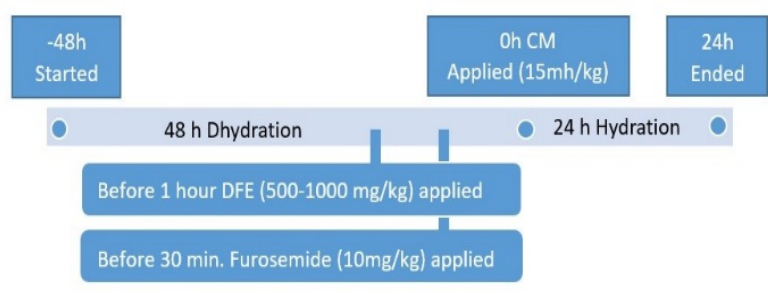

Figure 1. Experimental design diagram 


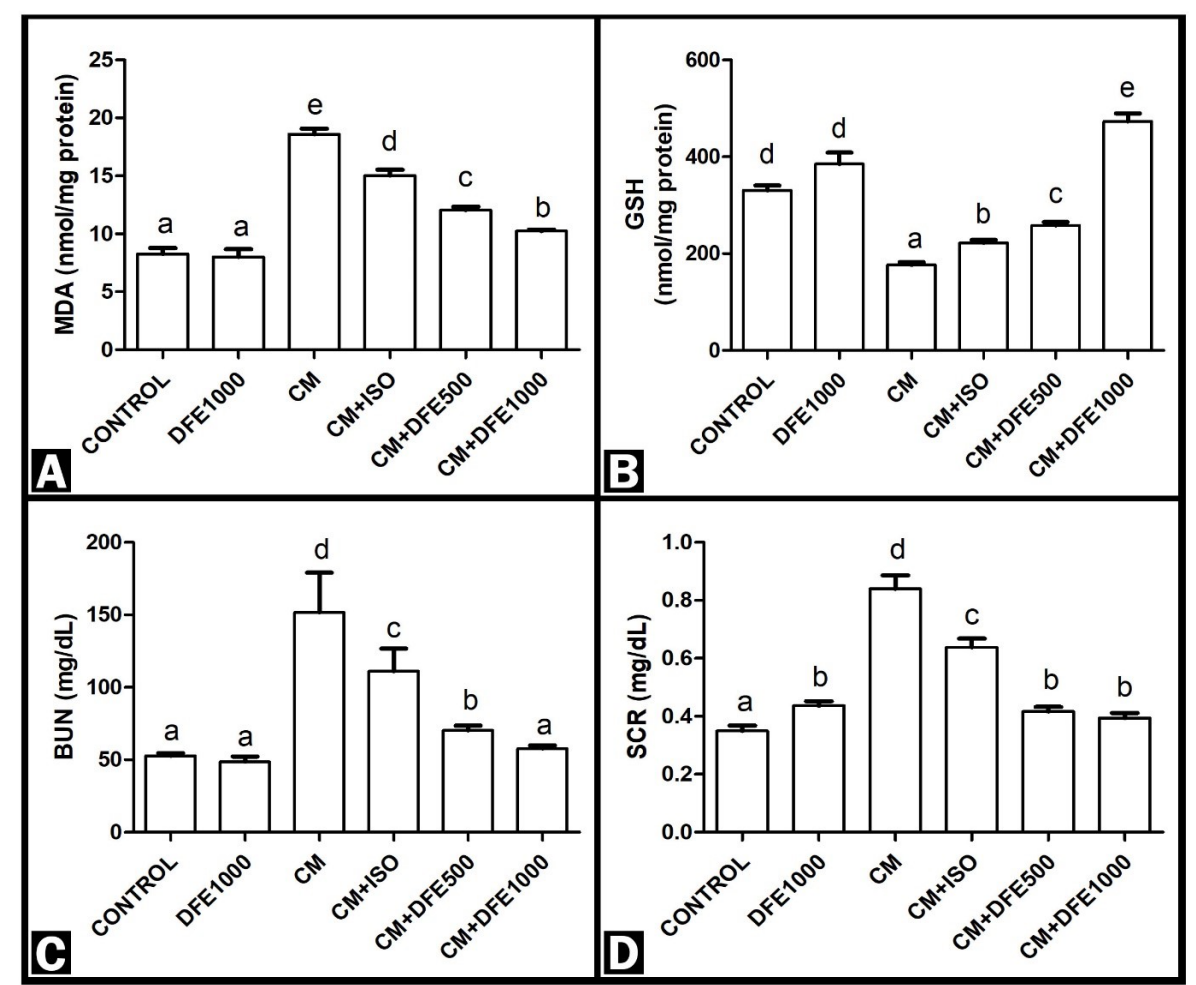

Figure 2. Biochemical analyses- A: MDA, B: GSH, C: BUN and D: SCR (BUN:23-74 mg/dL; SCR:0.6 mg/dL)(39).

In addition, furosemide was applied at the last 30 minutes of the dehydration period (30 minutes before CM application) (Figure 1). Then, CM (15 ml/kg) was given slowly from the tail vein to the other groups except the CONTROL and DFE1000 groups. Isotonic saline (ISO) $(3 \mathrm{ml} / \mathrm{kg})$ was administered by oral gavage in the last 1 hour of the 48-hour dehydration period to the CM+ISO group. DFE (500 and 1000 $\mathrm{mg} / \mathrm{kg}$ ) was administered by oral gavage in the last 1 hour of the 48-hour dehydration period (1 hour before $\mathrm{CM}$ administration) to the CM+ DFE500 and CM+DFE1000 groups. 24 hours after the application of $\mathrm{CM}$, the experiment was ended. At the end of the experiment, all animals were anaesthetized through the intraperitoneal administration of a combination of $15 \mathrm{mg} / \mathrm{kg}$ xylazine (Rompun, Bayer, Turkey) and $100 \mathrm{mg} / \mathrm{kg}$ ketamine (Ketalar, Phizer, Turkey). A longitudinal incision was created in the midline area of the lower abdomen. Kidney organs was observed was carefully keep via pens. Then, diaphragm was opened and the blood was taken directly from the heart through an injector into a gel tube. At the end of the experiment, animals were sacrificed by high dose thiopental sodium under anesthesia. Collected tissues were stored at $3.7 \%$ formaldehyde for histopathological and at $-80^{\circ} \mathrm{C}$ for biochemical examination. Blood samples were centrifuged at 4000 rmp for 10 minutes and the serum part was taken and stored at $-80{ }^{\circ} \mathrm{C}$. Histopathologists and lab data analysts were double blinded for each group.

\section{Biochemical Analyses \\ Kidney Fonctions Marker Analyses}

BUN and SCR analyses were performed using the by using kits compatible with Roche Modular autoanalyzer (cobas c-501, Roche Diagnostic, Germany), using photomotric methods (Principle of Urea test: based on the kinetic test with urease and glutamate dehydrogenase; Principle of SCR test: based on the kinetic calorimetric test. As a result of analyses, the units of serum BUN and SCR levels were given as $\mathrm{mg} / \mathrm{dL}$.

\section{Oxidative stress Marker Analyses}

For determination MDA and GSH levels that is oxidant/antioxidant parameters, $100 \mathrm{mg}$ of tissue was weighed for each animal. All tissue was homogenized in a homogenizer device. For biochemical studies, while MDA levels in supernatants were measured colorimetrically according to the methods reported by Yoshioko and his friends at 535nm (20), GSH levels are measured colorimetrically as regards of Beutler 
and his colleagues reports at $412 \mathrm{~nm}$ by invitrogen elisa reader (21). Biochemical analyses were carried out at Kafkas University, Central Research Laboratory.

\section{Histologic Analyses}

Kidney tissues were rapidly fixed in $3.7 \%$ solution formaldehyde for $48 \mathrm{hr}$. After fixation, all samples for histological tissue processing were routinely performed. According to histological tissue processing, all tissues were passed through increasing alcohol $(50,60,70,80,96,99-1$ hour) series. Subsequently, 3 series of xylene were kept in solution (3x15 minutes). Finally, it was kept in molten soft and hard liquid paraffin. At the end of the followup procedure, tissues were individually blocked in paraffin. After tissue processing, 5 micrometers thick sections were taken from each paraffin block for histopathological examination. Kidney tissue slides was stained with hematoxylin \& eosin.

\section{Statistical Analysis}

The data of our study was statistically evaluated with IBM 20.00 SPSS program. The groups were compared to Tukey's post-hoch tests from the oneway ANOVA multiple comparison test with a value of $P<0.05$ considered statistically significant.

\section{RESULTS}

\section{Renal Functions Markers \\ BUN analyze}

$\mathrm{CM}$ group was significantly increased compared to CONTROL and DFE1000 groups $(p<0.05)$. In CM ISO, CM+DFE500 and CM+DFE1000 groups were significantly decreased compared to the CM group ( $p$ $<0.05)$. CM+DFE500 and CM+DFE1000 groups were significantly reduced compared to the $\mathrm{CM}+\mathrm{ISO}$ group ( $p<0.05)$. In addition, In CM + DFE1000 group was observed a significant decrease $(p<0.05)$ compared to the $\mathrm{CM}+500$ group, while no significance was observed between the CM + DFE1000 and CONTROL groups ( $p>0.05$ ) (Figure $2 C$ ).

\section{Creatinine analyze}

A significant decrease was found in $\mathrm{CM}+\mathrm{ISO}$, CM+DFE500, CM+DFE1000 and CONTROL groups compared to the CM group ( $p<0.05)$. CM+DFE500, $\mathrm{CM}+$ DFE1000 groups were found to be significantly decreased compared to CM+ISO group $(p<0.05)$. However, there was no significant difference between the DFE1000, CM+ DFE500 and CM+DFE1000 groups $(p>0.05)$ (Figure 2D).

\section{Oxidative Stress Markers MDA analyze}

There was no significant difference between CONTROL group and DFE1000 group ( $p>0.05)$. CM group was significantly increased compared to CONTROL group (p <0.05). In CM+ISO, CM+DFE500, CM+DFE1000 groups were found to be significantly decreased compared to $\mathrm{CM}$ group ( $\mathrm{p}$ $<0.05)$. In CM+DFE500, CM+DFE1000 groups were found to have a significant decrease compared to $\mathrm{CM}+$ ISO group $(\mathrm{p}<0.05)$. There was also a significance between CM+DFE500 and CM+DFE1000 $(p<0.05)$ (Figure 2A).

\section{GSH analyze}

In CM+ISO, CM+DFE500, CM+DFE1000 groups were found to be significantly increased compared to CM group ( $p$ <0.05). In CM+DFE500, CM+DFE1000 groups showed a significant increase compared to CM+ISO group ( $p<0.05$ ). In addition, a significance between CM+DFE500 and CM+DFE1000 was observed $(p<0.05)$. There was a significant decrease in CM group compared to CONTROL group $(p<0.05)$. There was no significant difference between the CONTROL group and the DFE1000 group $(p>0.05)$ (Figure 2B).

\section{Histopathological Findings}

In the CONTROL group, no pathological finding was found in the glomerular, distal and proximal tubules (Figure 3A). The DFE1000 group was similar histological appearance to the healthy group (Figure 3B). While shrinkage was observed in the glomerular structures in the CM group, epithelial vacuolization was also observed in the distal and proximal tubules. In addition, inflammation was observed around the glomerular tangle and distal tubules (Figure 3C). While irregular distribution and enlargement were observed in the glomeruli in the $\mathrm{CM}+\mathrm{ISO}$ group, epithelial vacuolization was also observed in the distal and proximal tubules (Figure 3D). In the CM+DFE500 group was reduced epithelial vacuolization in the distal and proximal tubules compared to the CM group (Figure 3E). Similar to the CONTROL group, in the CM+DFE1000 group, epithelial vacuolization was decreased in the distal and proximal tubules compared to the $\mathrm{CM}$ group (Figure 3F). 


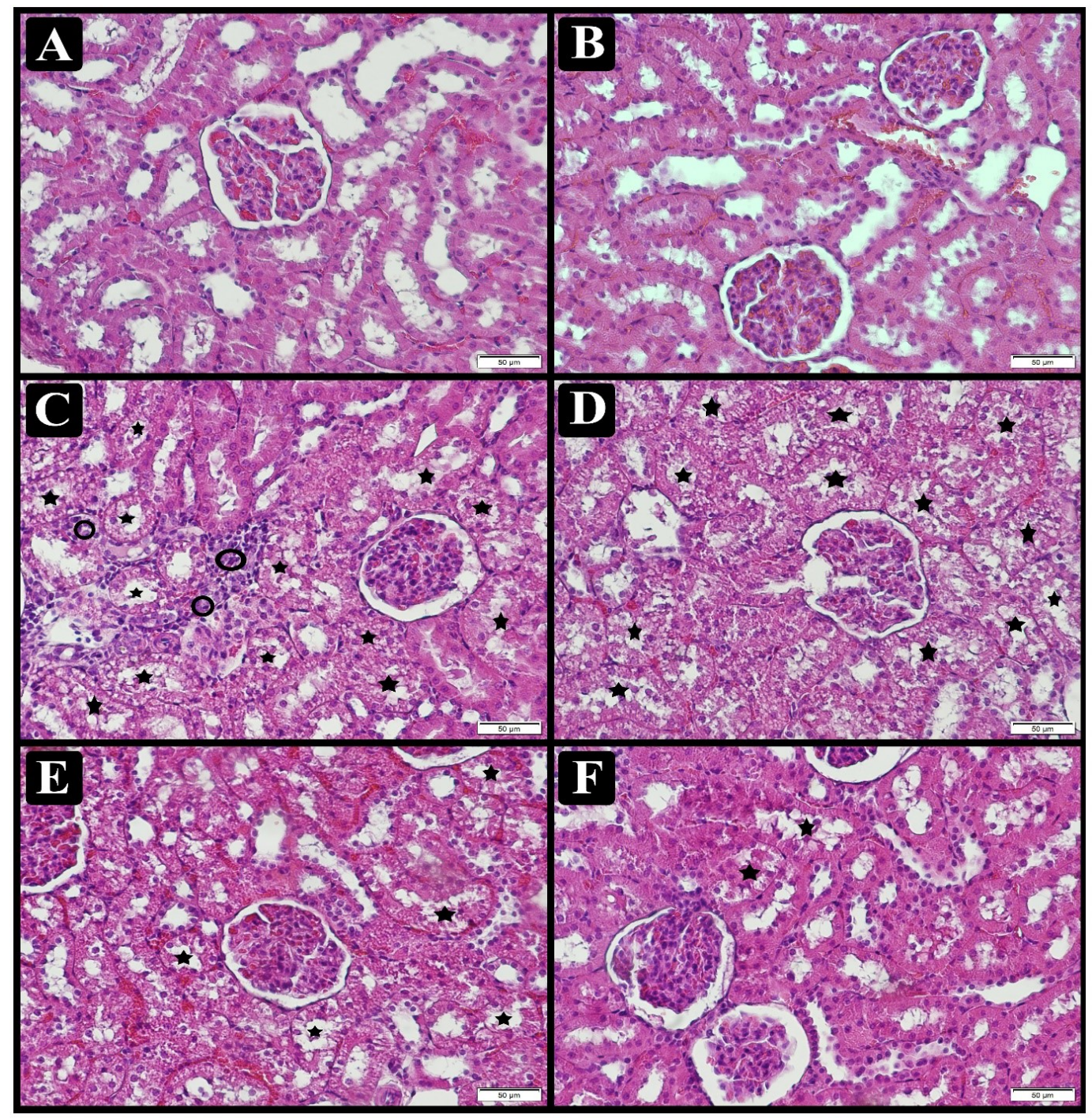

Figure 3. Histopathologic findings- A: Control group, B: DFE group, C: CM group, D: CM+ISO group, E: CM+DFE500 group, F: CM+DFE1000 group (Stars: Tubular vacuolization and damage, Circle: Inflammation area)

Morever to make the histopathological findings more understandable; tubular vacuolization and inflammation findings were semi-quantitatively scored as - (absent), + (mild), ++ (moderate), and +++ (severe) (Table 1).

\section{DISCUSSION}

Although the CIN rate in the community is estimated to be around $2 \%$, it can rise to $25 \%$ in the elderly, those with diabetes, acute coronary syndrome and chronic renal failure (22). CIN is also responsible $10 \%$ of AKI occurring in the hospital (6). CIN can prolonged hospital stay and increase treatment costs. It may be cause to irreversible terminal renal failure when treatment is delayed (23). Therefore, it is very important to use an anti-CIN agent. Treatment agents for CIN damage have anti-inflammatory and antioxidant properties.

Red dragon fruit has phytochemical bioactive compounds like polyphenols, flavonoids $(24,25)$. The fruit has also scavenging property against alkyl and hydroxyl radical (24). Because of this feature, it is able to assist with the balancing of oxidative stress. In one study, DFE was reported to increase MDA and GPX levels in oxidative damage (26). In this study, we investigated that in eliminating $\mathrm{CIN}$ damage role of DFE which its high antioxidant content. To our knowledge, nephropathy caused by radiocontrast will also be the first study to investigate the effects of DFE in preventing renal dysfunction. 


Table 1. Group description and histopathological scoring
\begin{tabular}{|l|l|l|}
\hline & Group Description & $\begin{array}{l}\text { Tubular } \\
\text { Vacuolization }\end{array}$ \\
\hline CONTROL & No substance was administered to this group. & - \\
\hline DFE1000 & Only applied DFE1000 mg/kg was administered & - \\
\hline CM & $\begin{array}{l}\text { Contrast media }(15 \mathrm{ml} / \mathrm{kg}) \text { was administered. } \\
\text { Contrast media }(15 \mathrm{ml} / \mathrm{kg})+0.9 \% \text { Isotonic saline }(15\end{array}$ & ++ \\
\hline CM+ISO & $\begin{array}{l}\text { Contrast media }(15 \mathrm{ml} / \mathrm{kg}) \text { and DFE } 500 \mathrm{mg} / \mathrm{kg} \text { was } \\
\text { administered }\end{array}$ & ++ \\
\hline CM+DFE500 & $\begin{array}{l}\text { Contrast media }(15 \mathrm{ml} / \mathrm{kg}) \text { and DFE } 1000 \mathrm{mg} / \mathrm{kg} \text { was } \\
\text { administered }\end{array}$ & ++ \\
\hline CM+DFE1000 & ++ \\
\hline
\end{tabular}

Parameters such as SCR and BUN can provide information about kidney pathophysiology. Indeed, these two parameters are the most important parameters used in the diagnosis of CIN. Studies found a significant increase in both BUN and SCR levels in CM groups compared to the control group $(27,28)$. In our study, we found that BUN and SCR levels increased in CM groups, while these levels decreased in DFE treatment groups. Our findings are similar to previous oxidative stress-antioxidant related studies $(28,29)$. In previous studies of this fruit, these two parameters have never been used. This strengthens the value of our article.

The most important mechanism underlying the pathogenesis of $\mathrm{CIN}$ is the presence of increased oxidative stress (30). Oxidative stress can cause from overproduction of reactive oxygen species (ROS) by metabolic reactions that use oxygen and changing balance of the oxidant/antioxidant statuses in favor of the oxidants (31).

After contrast is administered, increased ROS leads to lipid peroxidation and cytotoxic damage. MDA (end products of lipid peroxidation of membrane polyunsaturated fatty acids by free radicals) caused by lipid peroxidation is indicator of oxidative damage (32). Studies on CIN and antioxidant agents have shown an increase in MDA levels in kidney tissue in CIN groups $(28,32,33)$. In the Kongkham et al study, it was found that lipid peroxidation products increased in terms of MDA in both kidney tissue and serum (28). In parallel with these findings, MDA was found to be high in the CM group in our study. On the other hand, it was found that MDA levels decreased significantly in DFE administered groups. This finding reflects the DFE antioxidant content (34). Another important parameter for assessing oxidative damage to tissue is GSH (35). GSH, which acts as an intracellular antioxidant, is insufficient in cases of severe oxidative damage, such as ischemia, sepsis and CIN (36). Indeed, in our study, Levels of GSH decreased in CM groups. The work done by Buyuklu and his colleagues supports our study (37). In addition to, GSH levels increased in DFE treatment groups. This result suggests that DFE supports the intracellular antioxidant system. This result is consistent with the study showing that Dragon fruit increases GSH-Px activities and GSH levels (38).

The most obvious consequence of $\mathrm{CIN}$ is renal tubular vacuolization. Vacuolization is the result of pinocytic vesicles in the transition to $\mathrm{CM}$ renal tubules. This vacuolization is reversible and does not cause damage (39). Morever, exposure to CM in AKI may cause loss of renal function. In the literature, CM exposure has been shown to cause tubular damage via hypoxia by reducing renal blood flow or by CM's toxic effect on tubular cells (2). In our study, tubular vacuolization and subsequent tubular damage are observed in rats with AKI caused by CM application. Neutrophil infiltration resulting from cellular damage shows the severity of the damage in the CM group. On the other hand, the most clear finding showing decreased tubular damage is the absence of neutrophil infiltration, while decreased vacuolization was observed in the tubules in the DFE groups. In the light of all information, it has been shown that the DFE1000 group is a dose-dependent effect on mesenteric artery ischemia damage compared to the DFE500 group by histopathological, biochemical and molecular parameters.

$\mathrm{CM}$ application in AKI causes tubular damage due to oxidative stress in the kidneys. Only isotonic saline treatment is insufficient against the kidney damage. In our study, significant decreases were observed in BUN and creatinine values in groups given DFE. In addition, CM+DFE application has been shown to play a scavenging role against ROS. These results 
show that DFE application together with CM can play a protective role against renal tubule damage. Especially in the group given DFE1000, tubular vacuolization was significantly reduced. This effect of dragon fruit, whose popularity has increased in recent years, will make our study a reference source for new studies on this fruit in the future.

\section{Highlights of the Study}

One of the major causes of hospital-acquired acute kidney injury is contrast-induced nephropathy.

For the treatment of nephropathy damage, agents with anti-inflammatory and antioxidant properties are preferred.

Dragon fruit extract can be used as supportive and protective in this damage in tissue.

Abbreviations: AKI: Acute Kidney Injury, CIN: Contrast-Induced Nephropathy, DFE: Dragon Fruit Extract, CM: Contrast Media, BUN: Blood Urea Nitrogen, SCR: Serum Creatinine, GSH: Glutathione, MDA: Malondialdehyde, ISO: Isotonic saline, ROS: Reactive Oxygen Species

Acknowledgements: Thanks to Oğuzhan Kılıç for the fresh and fast delivery of the fruits.

Author Contributions: Concept - L.S., E.T.; Design - M.Y., A.H.; Supervision - P.A.K., H.F.G.; Resources - L.S., E.T.; Materials P.A.K, A.H., E.T.; Data Collection and/or Processing - L.S., E.T., H.F.G.; Analysis and/or Interpretation - M.Y., E.T.; Literature Search - L.S., E.T.; Writing Manuscript - L.S., E.T., M.Y.; Critical Review - L.S., M.Y., A.H.

Conflict of Interest: No conflict of interest was declared by the authors.

Ethics Committee Approval: Ethics committee approval was received for this study from the ethics committee of Kafkas University local ethics committee on animal experiments (2020/047)

Financial Disclosure: The authors declared that this study has received no financial support.

Peer-review: Externally peer-reviewed.

\section{REFERENCES}

1. Li PK, Burdmann EA, Mehta RL. World Kidney Day 2013: acute kidney injury-global health alert. American journal of kidney diseases: the official journal of the National Kidney Foundation 2013; 61(3): 359-63.

2. Itoh $\mathrm{Y}$, Yano $\mathrm{T}$, Sendo $\mathrm{T}$, Oishi R. Clinical and experimental evidence for prevention of acute renal failure induced by radiographic contrast media. Journal of pharmacological sciences 2005; 97(4): 473-88.

3. Jabara R, Gadesam RR, Pendyala LK, et al. Impact of the definition utilized on the rate of contrast-induced nephropathy in percutaneous coronary intervention. The American journal of cardiology 2009; 103(12): 1657-62.

4. Sendeski MM. Pathophysiology of renal tissue damage by iodinated contrast media. Clinical and experimental pharmacology \& physiology 2011; 38(5): 292-9.

5. Hizoh I, Haller C. Radiocontrast-induced renal tubular cell apoptosis: hypertonic versus oxidative stress. Investigative radiology 2002; 37(8): 428-34.

6. Heyman SN, Rosen S, Khamaisi M, Idee JM, Rosenberger C. Reactive oxygen species and the pathogenesis of radiocontrast-induced nephropathy. Investigative radiology 2010; 45(4): 188-95.

7. Faggioni M, Mehran R. Preventing Contrastinduced Renal Failure: A Guide. Interventional cardiology 2016; 11(2): 98-104.

8. Spargias K, Alexopoulos E, Kyrzopoulos S, et al. Ascorbic acid prevents contrast-mediated nephropathy in patients with renal dysfunction undergoing coronary angiography or intervention. Circulation 2004; 110(18): 2837-42.

9. Pannu N, Wiebe N, Tonelli M, Alberta Kidney Disease N. Prophylaxis strategies for contrastinduced nephropathy. Jama 2006; 295(23): 2765-79. Doi:10.1001/jama.295.23.2765

10. Marenzi G, Assanelli E, Marana I, et al. Nacetylcysteine and contrast-induced nephropathy in primary angioplasty. The New England journal of medicine 2006; 354(26): 2773-82.

11. Colbay M, Yuksel S, Uslan I, et al. Novel approach for the prevention of contrast nephropathy. Experimental and toxicologic pathology: official journal of the Gesellschaft fur Toxikologische Pathologie 2010; 62(1): 81-9.

12. Onbasili $A O$, Yeniceriglu $Y$, Agaoglu $P$, et al. Trimetazidine in the prevention of contrastinduced nephropathy after coronary procedures. Heart 2007; 93(6): 698-702.

13. Yoshida S, Kamihata $H$, Nakamura $S$, et al. Prevention of contrast-induced nephropathy by chronic pravastatin treatment in patients with cardiovascular disease and renal insufficiency. Journal of cardiology 2009; 54(2): 192-8.

14. Macias-Ceja DC, Cosin-Roger J, Ortiz-Masia D, et al. The flesh ethanolic extract of Hylocereus polyrhizus exerts anti-inflammatory effects and prevents murine colitis. Clinical nutrition 2016; 35(6): 1333-9. 
15. Wu L-C, Hsu H-W, Chen Y-C, Chiu C-C, Lin Y-I, Ho J-aA. Antioxidant and antiproliferative activities of red pitaya. Food Chemistry 2006; 95(2): 319-27

16. Zainoldin Kh, Baba AS. The Effect of Hylocereus Polyrhizus and Hylocereus Undatus On Physicochemical, Proteolysis, And Antioxidant Activity In Yogurt. World Academy of Science, Engineering and Technology 2009;60:361-6

17. Castro-Enriquez DD, Montano-Leyva B, Del Toro-Sanchez CL, et al. Effect of Ultrafiltration of Pitaya Extract (Stenocereus thurberi) on Its Phytochemical Content, Antioxidant Capacity, and UPLC-DAD-MS Profile. Molecules 2020; 25: 2.

18. Hartono MR, Suardita K, Yuliati A. Proliferation and osteogenic differentiation of bone marrowderived mesenchymal stem cell after exposure to red flesh dragon fruit extract. Dental research journal 2020; 17(2): 107-13

19. Som AM, Ahmat N, Abdul Hamid HA, Azizuddin N. A comparative study on foliage and peels of Hylocereus undatus (white dragon fruit) regarding their antioxidant activity and phenolic content. Heliyon 2019; 5(2): e01244.

20. Yoshioka T, Kawada K, Shimada T, Mori M. Lipid peroxidation in maternal and cord blood and protective mechanism against activated-oxygen toxicity in the blood. American journal of obstetrics and gynecology 1979; 135(3): 372-6.

21. Beutler E, Duron O, Kelly BM. Improved method for the determination of blood glutathione. The Journal of laboratory and clinical medicine 1963; 61: 882-8

22. Akgullu C, Hekim T, Eryilmaz U, et al. The usefulness of carvedilol and nebivolol in preventing contrast nephropathy in rats. Renal failure 2015; 37(3):511-7.

23. Rihal CS, Textor SC, Grill DE, et al. Incidence and prognostic importance of acute renal failure after percutaneous coronary intervention. Circulation 2002; 105(19): 2259-64.

24. Kim H, Choi HK, Moon JY, Kim YS, Mosaddik A, Cho SK. Comparative antioxidant and antiproliferative activities of red and white pitayas and their correlation with flavonoid and polyphenol content. Journal of food science 2011; 76(1): C38-45.

25. Morais SGG, da Silva Campelo Borges G, Dos Santos Lima M, Martin-Belloso O, Magnani M. Effects of probiotics on the content and bioaccessibility of phenolic compounds in red pitaya pulp. Food research international 2019; 126: 108681.

26. Harahap NS, Sinaga FA, Sinaga RN, Amelia R. Effect of Red-Fleshed Pitaya (Hylocereus Polyrhizus) to Increase Gluthatione Peroxidase Levels in Male Rats (Rattus Norvegicus): The Induced Oxidative Stress. The 5th Annual International Seminar on Trends in Science and Science Education, Medan, Indonesia, 2018.

27. Boyacioglu M, Turgut $\mathrm{H}$, Akgullu C, Eryilmaz U, Kum C, Onbasili OA. The effect of L-carnitine on oxidative stress responses of experimental contrast-induced nephropathy in rats. The Journal of veterinary medical science 2014; 76(1): 1-8.

28. Kongkham S, Sriwong S, Tasanarong A. Protective effect of alpha tocopherol on contrastinduced nephropathy in rats. Nefrologia: publicacion oficial de la Sociedad Espanola Nefrologia 2013; 33(1): 116-23.

29. Aksu F, Aksu B, Unlu N, et al. Antioxidant and renoprotective effects of sphingosylphosphorylcholine on contrastinduced nephropathy in rats. Renal failure 2016; 38(7): 1089-98.

30. Aspelin P, Aubry P, Fransson SG, et al. Nephrotoxic effects in high-risk patients undergoing angiography. The New England journal of medicine 2003; 348(6): 491-9.

31. Birben E, Sahiner UM, Sackesen C, Erzurum S, Kalayci O. Oxidative stress and antioxidant defense. The World Allergy Organization journal 2012; 5(1): 9-19.

32. Ari E, Kedrah AE, Alahdab Y, et al. Antioxidant and renoprotective effects of paricalcitol on experimental contrast-induced nephropathy model. The British journal of radiology 2012; 85(1016): 1038-43.

33. Toprak O, Cirit M, Tanrisev M, et al. Preventive effect of nebivolol on contrast-induced nephropathy in rats. Nephrology, dialysis, transplantation: official publication of the European Dialysis and Transplant Association European Renal Association 2008; 23(3): 853-9.

34. Rebecca O, Boyce A, Chandran S. Pigment identification and antioxidant properties of red dragon fruit (Hylocereus polyrhizus). African Journal of Biotechnology 2010; 9(10): 1450-4 
35. Jones DP. Radical-free biology of oxidative stress. American journal of physiology Cell physiology 2008; 295(4): C849-68.

36. Ho E, Karimi Galougahi K, Liu CC, Bhindi R, Figtree GA. Biological markers of oxidative stress: Applications to cardiovascular research and practice. Redox biology 2013; 1: 483-91.

37. Buyuklu M, Kandemir FM, Ozkaraca M, Set T, Bakirci EM, Topal E. Protective effect of curcumin against contrast induced nephropathy in rat kidney: what is happening to oxidative stress, inflammation, autophagy and apoptosis? European review for medical and pharmacological sciences 2014; 18(4): 461-70

38. Vijayakumar R, Abd Gani SS, Zaidan UH, Halmi MIE, Karunakaran T, Hamdan MR. Exploring the Potential Use of Hylocereus polyrhizus Peels as a Source of Cosmeceutical Sunscreen Agent for Its Antioxidant and Photoprotective Properties. Evidence-based complementary and alternative medicine: eCAM 2020; 020: 7520736.

39. Kiss N, Hamar P. Histopathological Evaluation of Contrast-Induced Acute Kidney Injury Rodent Models. BioMed research international 2016; 2016: 3763250. 